\section{Defining an acceptable period of time from melanoma biopsy to excision}

\author{
Laura S. Huff, ${ }^{1}$ Caroline A. Chang, ${ }^{2}$ \\ Jacob F. Thomas, ${ }^{3}$ \\ Margaret Cook-Shimanek, ${ }^{4}$ \\ Paul Blomquist, ${ }^{5}$ \\ Nellie Konnikov, ${ }^{6}$ \\ Robert P. Dellavalle ${ }^{7,8,9}$ \\ 'University of Colorado School of \\ Medicine, Aurora, CO; ${ }^{2}$ Department of \\ Dermatology, Tufts Medical Center, \\ Boston, MA; ${ }^{3}$ Colorado School of Public \\ Health, Aurora, CO; ${ }^{4}$ Department of \\ Preventive Medicine, University of \\ Colorado, Aurora, CO; ${ }^{5} \mathrm{Health}$ Sciences \\ Library, University of Colorado, Aurora, \\ CO; ${ }^{\circ}$ Dermatology Service, Department \\ of Veterans Affairs Medical Center, \\ Boston, MA; ${ }^{7}$ Department of \\ Dermatology, University of Colorado, \\ Aurora, CO; ${ }^{8}$ Dermatology Service, \\ Department of Veterans Affairs Medical \\ Center, Denver, CO; ${ }^{9}$ Colorado School of \\ Public Health Department of \\ Epidemiology, Aurora, CO, USA
}

\section{Abstract}

Melanoma is the most lethal form of skin cancer and it is the second most common cancer among adolescents and young adults. The aim of this work is to determine if surgical intervals differ between four different clinics and between departments within the hospitals, and to compare these to industry standards. Surgical intervals were measured through retrospective chart review at four dermatology clinics. Of 205 melanoma cases, clinic and departmental median surgical intervals ranged 15-36.5 days and 26-48 days, respectively. There was significant association between clinic and time between biopsy and pathology report, time between pathology report and excision, and total surgical interval $(\mathrm{P}<0.0001$, $\mathrm{P}=0.03$, and $\mathrm{P}<0.0001$ respectively). There was significant association between department and time between pathology report and excision, and surgical interval $(\mathrm{P}<0.0001$, and $\mathrm{P}=0.003$ respectively). Pair-wise comparisons detected significantly longer intervals between some clinics and departments (maximum difference $67.3 \%, \mathrm{P}<0.0001$ ). Hypothesis-based, informal guidelines recommend treatment within 4-6 weeks. In this study, median surgical intervals varied significantly between clinics and departments, but nearly all were within a 6-week frame.

\section{Introduction}

Melanoma, the most lethal form of skin cancer, is expected to claim 8,790 lives in the United States in 2011. ${ }^{1}$ During a lifetime, 1/37 men and 1/55 women will develop melanoma. ${ }^{1}$ It is the second most common cancer among adolescents and young adults. ${ }^{2}$ While the incidences of most cancer types are declining, melanoma is one of the few cancers where incidence continues to climb in the United States. $^{3-5}$

Most melanoma (84\%) is diagnosed early as localized disease, which carries a high five year survival rate of $98 \% .{ }^{3}$ Advanced disease is associated with a devastating prognosis. ${ }^{5}$ Despite advances in adjuvant therapy, five year survival for distant disease is only $15 \%{ }^{3}$ Compared to other malignancies, melanoma is refractory to chemotherapy as well as newly evolving immunotherapies. ${ }^{5}$ Mainstays of treatment include early detection and surgical excision. ${ }^{1,6,7}$ Biopsy is performed for a suspicious skin lesion, and diagnosis is made by histological examination of tissue. If biopsy reveals melanoma, the primary tumor and surrounding tissue are removed with surgical excision; sentinel lymph node biopsy is used for staging if the melanoma has significant thickness or histological characteristics. Metastatic disease is treated with further lymph node surgery, immunotherapy, chemotherapy, and radiation therapy; yet these provide minimal benefit. Many sufferers of metastatic disease are encouraged to enroll in clinical trials for experimental treatment because the prognosis is so guarded. ${ }^{1}$

Increased risk of metastasis is associated with ulceration (bleeding) and depth of tumor. The strongest predictor for survival is having regional lymph nodes that are free from metastasis. ${ }^{8-10}$ Early diagnosis and treatment are considered paramount for melanoma, and delayed treatment is hypothesized to affect survival. ${ }^{6,11}$ In the 1980's, a Scottish study revealed a surprisingly high proportion of patients presenting with melanomas categorized as thick, poor prognosis and only $16 \%$ of patients underwent surgical treatment within three months of noticing the pigmented lesion. In response, a public education campaign began to promote early detection. Within six months, the percentage of patients presenting with melanomas categorized as thin, good prognosis increased (38\% to 62\%) and thick, poor prognosis decreased (34\% to $15 \%){ }^{12}$

Many factors contribute to treatment delays such as time it takes for patients to notice lesions, obtain appointments/referrals, receive biopsies, obtain biopsy results, and schedule treatment. Specifically, the time between diagnostic biopsy and surgical excision, known as
Correspondence: Robert P. Dellavalle, Department of Veteran Affairs Medical Center, 1055 Clermont Street, Box 165, Denver, C0 80220, USA.

Tel. +1.303.399.8020-2475 - Fax: +1.303.393.4686. E-mail: robert.dellavalle@ucdenver.edu

Key words: melanoma, surgical interval, treatment time, melanoma survival, time factors.

Acknowledgements: statistical analysis was funded by the University of Colorado School of Medicine and was performed by the Research Consulting Laboratory at the Colorado School of Public Health. This study was partially funded by the U.S. Department of Veterans Affairs (Dr. Robert Dellavalle). The authors thank Emilee Sandsmark for editing.

Contributions: LSH, coordinated efforts, gathered data, helped with statistical analysis, and wrote the manuscript; CAC, gathered data; JFT, performed the statistical analysis and helped write the manuscript; MCS helped write and revise the manuscript; $\mathrm{PB}$ performed a literature search and helped write the manuscripts; NK gathered data and provided project oversight; RPD acted as principal investigator, gathered data, and provided project oversight.

Conflicts of interest: the authors have no conflict of interest to declare.

Received for publication: 26 August 2011. Revision received: 4 November 2011. Accepted for publication: 18 November 2011.

This work is licensed under a Creative Commons Attribution NonCommercial 3.0 License (CC BYNC 3.0).

(C) Copyright L.S. Huff et al., 2012

Licensee PAGEPress, Italy

Dermatology Reports 2012; 4:e2

doi:10.4081/dr.2012.e2

the surgical interval, is a modifiable factor that may be impacting melanoma outcomes.

\section{Materials and Methods}

\section{Objective}

The aim of this work is to determine if surgical intervals differ between four different clinics and between departments within the hospitals, and to compare these to industry standards.

\section{Design}

Surgical intervals were measured through quality improvement projects at four dermatology clinics (Veterans Affairs Dermatology 
Clinic, Denver, Colorado; Veterans Affairs Dermatology Clinic, Boston, Massachusetts; University of Colorado Hospital Dermatology Clinic, Aurora, Colorado; University of Colorado Hospital, Melanoma Clinic, Aurora, Colorado). Retrospective chart reviews were conducted for all patients having a histological diagnosis of melanoma during a one year time interval. Days between biopsy and pathology results (time to diagnosis), pathology results and surgical excision (time to surgery), and surgical interval time (time to diagnosis + time to surgery) were measured. The department performing surgical excision was documented (Dermatology, General Surgery, MOHS, or Plastic Surgery). Not all departments were present at each hospital. This research was approved by the Colorado Multiple Institutional Review Board (Protocol 10-1338).

\section{Statistical analysis}

A two-way ANOVA (analysis of variance) was used to test for all differences between clinics performing biopsies and departments performing surgeries for three outcomes (time to diagnosis, time to surgery, surgical interval). An interaction between department and clinic was tested for all three outcomes to determine if the time difference in means between each clinic significantly changed depending on department, or if the difference in means between each department changed depending on clinic. Since the interaction term for all three outcomes was non-significant, it was taken out of the analysis.

Within the framework of the two-way ANOVA model, all pair-wise comparisons were tested between each clinic for all three outcomes, and between each department for all three outcomes. P-values were adjusted using the Tukey adjustment for multiple comparisons.

Outcomes were log-transformed for the ANOVA due to violations of the normality assumption; therefore, results must be inter- preted on a multiplicative scale. Medians were used for summary statistics instead of means because of heavy right skew of data. Two departments (MOHS and Plastic Surgery) were not present at all hospitals and had insufficient amount of data. Therefore, these departments were excluded and not aggregated into the clinic data or included in the ANOVA analyses. Summary statistics for MOHS and Plastic Surgery are reported separately, as well as results showing the impact of removing these departments from aggregated clinic data.

\section{Results}

A total of 205 melanoma cases were identified among the four institutions and included in this study. Median surgical intervals within clinics ranged from 15 to 36.5 days and from 26 to 48 days within departments performing excisions (Table 1). Patients diagnosed in the University Hospital Dermatology Clinic had the shortest median surgical interval and the Denver Veteran's Affairs Clinic had the longest (15 and 36.5 days respectively). Among departments performing excisions, MOHS had the shortest median surgical interval and Plastic Surgery had the longest (26 and 48 days respectively).

Excluding MOHS and Plastic Surgery cases from the aggregated clinic data did affect the summary statistics shown in Table 1. The median time to diagnosis, time to surgery, and surgical interval at the University Hospital Dermatology Clinic all decreased from 5, 17, and 23.5 days respectively (MOHS included) to $4.5,10$, and 15 days respectively (MOHS excluded). The median time to diagnosis, time to surgery, and surgical interval at the Boston Veterans Affairs Dermatology Clinic changed from 9, 27, and 38 days respectively (Plastic
Surgery included) to 10,22 , and 31 days respectively (Plastic Surgery excluded).

There was a significant association between clinic and time between biopsy and pathology report (time to diagnosis), time between pathology report and surgical excision (time to surgery), and surgical interval $(\mathrm{P}<0.0001$, $\mathrm{P}=0.03$, and $\mathrm{P}<0.0001$ respectively). There was also a significant association between department performing surgery and time to surgery and surgical interval $(\mathrm{P}<0.0001$ and $\mathrm{P}=0.003$ respectively), but not time to diagnosis $(\mathrm{P}=0.30)$.

Certain clinics and departments had significantly shorter intervals during pair-wise comparisons (Table 2). For time to diagnosis, most notable was the 0.327 times shorter $(67.3 \%$ shorter) interval at the University Hospital Melanoma Clinic compared to the Denver Veterans Affairs Dermatology Clinic (95\% CI 0.194-0.551, $\mathrm{P}<0.0001)$. There was no statistical difference in time to diagnosis when comparing departments that performed the surgical excision.

Time to surgery was 0.414 times shorter (58.6\% shorter) at the University Hospital Dermatology Clinic compared to the Boston Veteran's Affairs Dermatology Clinic (95\% CI $0.182-0.938, \mathrm{P}=0.029)$. Other clinic pair-wise comparisons did not reach statistical significance for time to surgery. Dermatology departments had time to surgery that was 0.581 times shorter ( $41.9 \%$ shorter) than General Surgery departments (95\% CI 0.445-0.758, $\mathrm{P}<0.0001)$.

Surgical intervals were significantly shorter at the University Hospital Dermatology and Melanoma Clinics compared to both the Boston and Denver Veteran's Affairs Dermatology Clinics (ranging 33.9\% to 60.1\%). Surgical intervals were also significantly shorter depending on which department performed the excisions, with the Dermatology Clinic being $26.5 \%$ shorter than General Surgery (95\% CI 0.602-0.897, $\mathrm{P}=0.003)$.

Table 1. Surgical interval summary by dermatology clinic and surgical department.

\begin{tabular}{|c|c|c|c|c|c|c|}
\hline Category & $\begin{array}{l}\text { Time to } \\
\text { median }\end{array}$ & $\begin{array}{l}\text { agnosis (days)* } \\
\text { range/standard } \\
\text { deviation } / \mathbf{n}\end{array}$ & $\begin{array}{l}\text { Time to } \\
\text { median }\end{array}$ & $\begin{array}{l}\text { surgery (days) } \\
\text { range/standard } \\
\text { deviation/n }\end{array}$ & $\begin{array}{l}\text { Surgice } \\
\text { median }\end{array}$ & $\begin{array}{l}\text { interval (days) } \\
\text { range/standard } \\
\text { deviation/n }\end{array}$ \\
\hline \multicolumn{7}{|l|}{ Clinic } \\
\hline Veteran's affairs - Boston & 10 & $0-59 / 15.4 / 46$ & 22 & $1-119 / 23.4 / 47$ & 31 & $5-172 / 32.9 / 45$ \\
\hline Veteran's affairs - Denver & 19.5 & $1-38 / 9.6 / 22$ & 22.5 & $3-63 / 18.7 / 20$ & 36.5 & $7-101 / 19.8 / 20$ \\
\hline University Hospital - Melanoma clinic & 4 & $0-22 / 4.3 / 92$ & 22.5 & 0-70/15.7/92 & 26.5 & $4-79 / 16.7 / 92$ \\
\hline University Hospital - Dermatology clinic & 4.5 & $3-19 / 6.3 / 10$ & 10 & $3-24 / 7.4 / 7$ & 15 & $8-28 / 7.9 / 7$ \\
\hline \multicolumn{7}{|l|}{ Department } \\
\hline Plastic surgery & 5 & $2-48 / 11.4 / 15$ & 42 & $17-78 / 18.5 / 17$ & 48 & 21-90/21.1/15 \\
\hline General surgery & 5.5 & $0-38 / 6.3 / 80$ & 25 & $0-69 / 15.0 / 80$ & 31 & $4-101 / 17.4 / 80$ \\
\hline Dermatology & 7 & $0-59 / 13.1 / 85$ & 15.5 & $0-119 / 20.9 / 86$ & 28 & $5-172 / 28.1 / 84$ \\
\hline Micrographic surgery (MOHS) & 5 & $1-15 / 3.7 / 17$ & 20 & $2-72 / 18.4 / 17$ & 26 & $4-78 / 17.9 / 17$ \\
\hline
\end{tabular}

*Time to diagnosis is defined as the days between biopsy and pathology results. ${ }^{\circ}$ Time to surgery is defined as days between pathology results and surgical excision. ${ }^{*}$ The surgical interval is defined as the sum of the time to diagnosis and time to surgery. 
Table 2. Statistically significant pair-wise comparisons of specific clinics and departments by time to diagnosis, time to surgery, and surgical interval (All other pair-wise comparisons were not statistically significant).

\begin{tabular}{|c|c|c|c|c|c|}
\hline \multicolumn{2}{|c|}{ Pair-wise comparison } & Estimate & \multicolumn{2}{|c|}{$95 \% \mathrm{CI}$} & P-value* \\
\hline \multicolumn{6}{|l|}{ Time to diagnosis } \\
\hline University Hospital - Melanoma clinic & Veteran's affairs - Boston & 0.435 & 0.285 & 0.665 & $<.0001$ \\
\hline University Hospital - Dermatology clinic & Veteran's affairs - Denver & 0.362 & 0.145 & 0.901 & 0.022 \\
\hline University Hospital - Melanoma clinic & Veteran's affairs - Denver & 0.327 & 0.194 & 0.551 & $<0.0001$ \\
\hline \multicolumn{6}{|l|}{ Time to surgery } \\
\hline University Hospital - Dermatology clinic & Veteran's affairs - Boston & 0.414 & 0.182 & 0.938 & 0.029 \\
\hline Dermatology & General surgery & 0.581 & 0.445 & 0.758 & $<0.0001$ \\
\hline \multicolumn{6}{|l|}{ Surgical interval } \\
\hline University Hospital - Melanoma clinic & Veteran's affairs - Boston & 0.661 & 0.488 & 0.897 & 0.003 \\
\hline University Hospital - Melanoma clinic & Veteran's affairs - Denver & 0.635 & 0.437 & 0.921 & 0.01 \\
\hline University Hospital - Dermatology clinic & Veteran's affairs - Boston & 0.416 & 0.225 & 0.768 & 0.002 \\
\hline University Hospital - Dermatology clinic & Veteran's affairs - Denver & 0.399 & 0.208 & 0.766 & 0.002 \\
\hline Dermatology & General surgery & 0.735 & 0.602 & 0.897 & 0.003 \\
\hline
\end{tabular}

${ }^{*}$ All P-values were adjusted using the Tukey adjustment for multiple comparisons.

\section{Discussion}

Surgical intervals for some cancers are associated with survival outcomes and guidelines are defined. For instance, breast cancer treatment is recommended within 12 weeks of presentation, and longer delay is significantly associated with higher stage and worsened survival. ${ }^{10,13,14}$ Improved rectal cancer outcomes are associated with early resection and treatment is recommended within eight weeks. ${ }^{15}$ In contrast, colon cancer outcomes are not worse with delayed treatment, even up to 180 days. ${ }^{15}$

Surgical interval guidelines for melanoma are not well-defined and few known studies evaluate melanoma surgical intervals. One Scottish study found no association between surgical intervals ( $\leq 14$ days, 15-28 days, 29-42 days, 43-91 days, versus $\geq 92$ days) and overall survival, disease-free survival, or recurrencefree survival ( $\mathrm{P}=0.88,0.44,0.084$ respectively). The mean surgical interval of 986 patients was 30 days (range 1-468 days). ${ }^{16}$

A Mayo Clinic study similarly found no association between overall survival or diseasefree survival and surgical intervals of $\leq 28$ days versus $>28$ days. Overall survival and diseasefree survival were worse for patients with intervals $\geq 56$ days compared to $\leq 56$ days, but this difference did not reach statistical significance. The mean surgical interval of 473 patients was 30.5 days (median 27 days, range 0-244 days, standard deviation 20.4) with only $8 \%$ of patients having a surgical interval longer than 56 days. This study had significant limitations. The association between overall survival and disease-free survival with SLN status was significant in some, but not all statistical analyses models (all $\mathrm{P}<0.06$ ); ulceration was statistically significant for some, but not all statistical models evaluating overall survival $(\mathrm{P}<0.06)$; lesion thickness was statistically significant for disease-free survival but not for overall survival. Since SLN status, ulceration, and lesion thickness are well-accepted as the strongest predictors for melanoma outcomes, ${ }^{9}$ this raises concern about the power of the study. In addition, the median follow-up time was 2.8 years and only $20 \%$ of patients had follow up periods of 5 years or longer. ${ }^{10}$

A study from the California Tumor Registry compared survival rates of patients receiving biopsy with definitive surgery up to one week later versus immediate surgery without prior biopsy. There was no harm from delaying surgery. ${ }^{17}$

In contrast to the findings of the preceding studies, a retrospective study examining the United Kingdom (UK) two-week rule for referral of suspected cancer, found that providing patients who have pigmented lesions suspicious for melanoma with specialty care within two weeks of referral by their general practitioner (GP) was associated with reduced tumor thickness and improved survival. The authors compared waiting times and survival outcomes for patients who received specialist consultation and treatment at a rapid-access Pigmented Lesion Clinic (PLC) to those with traditional referral to outpatient plastic surgery. For all patients referred to the rapidaccess clinic $(n=4399)$ over a four year period, the delay between GP referral and PLC visit ranged from 0-14 days in contrast to patients seen during a two year period prior to PLC inception ( $n=756)$, where the waiting time for consultation ranged from 3-37 days. Of the 96 melanomas diagnosed in the PLC period, $96 \%$ had excisional biopsy within two weeks of their GP referral, with the majority (76\%) having their surgery on the day of PLC attendance. For melanoma patients seen in outpatient plastic surgery, the waiting time from GP referral to treatment ranged from 4 to 74 days (mean of 22.4 days). Melanomas diagnosed in the PLC within the 2-week time frame were significantly thinner than those diagnosed in the control group (mean tumor thickness of $1.68 \mathrm{~mm}$ versus $2.39 \mathrm{~mm}$, respectively; $\mathrm{P}<0.001)$. The five-year survival rates for patient treated in the PLC versus control groups were $82 \%$ and $62 \%$, respectively. Kaplan-Meier survival curves demonstrated a significantly improved survival for patients seen within two weeks compared with the control group (log rank chi-square 18.1924; $\mathrm{P}<0.001)$. The authors note that while it appears there is an evidence base for seeing patients with suspicious pigmented lesions within two weeks, the provider must additionally offer prompt surgical treatment. ${ }^{18}$

Longer follow-up and further research are needed before definitive conclusions can be made. In the current study, median surgical intervals varied significantly between departments and institutions. Despite this variance, nearly all institutions and departments had median surgical intervals similar to the mean surgical intervals (30 and 30.5 days) measured in the existing literature. ${ }^{10,16}$

\section{Limitations}

This was a descriptive study and no conclusions can be made about impact on survival or other outcomes. The sample size was small, which did not allow for ANOVA analyses of MOHS and Plastic Surgery cases. Data was collected from internal quality improvement projects at each institution, and there could be minor variations in how each institution collected data.

Known factors associated with longer surgical intervals are older age, male gender, and outside referral source, ${ }^{10}$ and these likely varied among the measured institutions although they were not directly measured. Other potential variable factors are race/ethnicity, socioeconomic status, and reliance of follow-up. 
Pathology reports were not accessed, but the severity of cases could have influenced surgical intervals. This study is geographically limited to Colorado and Massachusetts and did not evaluate the private sector or community health centers directly. However, the University Melanoma Clinic did receive a significant number of referrals from the private sector. This study did not investigate wait times for patients to attain appointments, which also significantly contributes to treatment delay.

\section{Conclusion}

It is unclear what surgical intervals affect melanoma outcomes. Two limited studies suggest that definitive treatment for melanoma should be offered in a timely manner, but does not need to be immediate..$^{10,16}$ Other evidence, however, suggests a more favorable tumor thickness and improved survival in those patients seen within two weeks of discovering a pigmented lesion, noting that benefit is maximized when accompanied by prompt surgical management. ${ }^{18}$ Hypothesis-based, informal guidelines from expert opinion recommend treatment within 4-6 weeks, although there is also evidence to suggest treatment within two weeks may improve survival. ${ }^{10,18}$ At the four institutions measured in this study, the median surgical intervals varied significantly between institutions and departments, but nearly all were within a 6 -week time frame. Available evidence suggests that more timely evaluation and surgical management of melanoma at the four institutions studied may contribute to improved disease outcomes.

\section{References}

1. American Cancer Society. Cancer Facts \& Figures 2011. Available from: http://www. cancer.org/Research/CancerFactsFigures/i ndex

2. National Cancer Institute. Epidemiology in Older Adolescents and Young Adults 15 to 29 Years of Age, Including SEER Incidence and Survival: 1975-2000. Available from: seer.cancer.gov/publications/aya/aya_mon o_complete.pdf

3. National Cancer Institute. SEER Cancer Statistics Review, 1975-2006. Available from: http://seer.cancer.gov/csr/1975_2006/

4. Jemal A, Siegel R, Ward E, et al. Cancer statistics, 2008. CA Cancer J Clin 2008;58 71-96.

5. Mouawad R, Sebert M, Michels J, et al. Treatment for metastatic malignant melanoma: old drugs and new strategies. Crit Rev Oncol Hematol 2010;74:27-39.

6. Brenner S, Tamir E. Early detection of melanoma: the best strategy for a favorable prognosis. Clin Dermatol 2002;20: 203-11.

7. Yao K, Balch G, Winchester DJ. Multidisciplinary treatment of primary melanoma. Surg Clin North Am 2009;89: 267-81.

8. Balch CM, Buzaid AC, Soong SJ, et al. Final version of the American Joint Committee on Cancer staging system for cutaneous melanoma. J Clin Oncol 2001;19:3635-48.

9. Balch CM, Soong SJ, Gershenwald JE, et al. Prognostic factors analysis of 17,600 melanoma patients: validation of the American Joint Committee on Cancer melanoma staging system. J Clin Oncol 2001;19:3622-34.
10. Carpenter S, Pockaj B, Dueck A, et al. Factors influencing time between biopsy and definitive surgery for malignant melanoma: do they impact clinical outcome? Am J Surg 2008;196:834-43.

11. Coldiron B. No evidence to support delay in excision of malignant melanoma. Arch Dermatol 2000;136:1269-71.

12. Doherty V, MacKie R. Reasons for poor prognosis in British patients with cutaneous malignant melanoma. Br Med J (Clin Res Ed) 1986;292:987-9.

13. Richards MA, Westcombe AM, Love SB, et al. Influence of delay on survival in patients with breast cancer: a systematic review. Lancet 1999;353:1119-26.

14. Richards MA, Smith P, Ramirez AJ, et al. The influence on survival of delay in the presentation and treatment of symptomatic breast cancer. Br J Cancer 1999;79: $858-64$.

15. Korsgaard M, Pedersen L, Sørensen HT, Laurberg S. Delay of treatment is associated with advanced stage of rectal cancer but not of colon cancer. Cancer Detect Prev 2006;30:341-6.

16. McKenna D, Lee R, Prescott R, Doherty V. The time from diagnostic excision biopsy to wide local excision for primary cutaneous malignant melanoma may not affect patient survival. Br J Dermatol 2002;147: 48-54.

17. Epstein E, Bragg K, Linden G. Biopsy and prognosis of malignant melanoma. JAMA 1969;208:1369-71.

18. Pacifico M, Pearl R, Grover R. The UK Government two-week rule and its impact on melanoma prognosis: an evidencebased study. Ann R Coll Surg Engl 2007;89:609-15. 